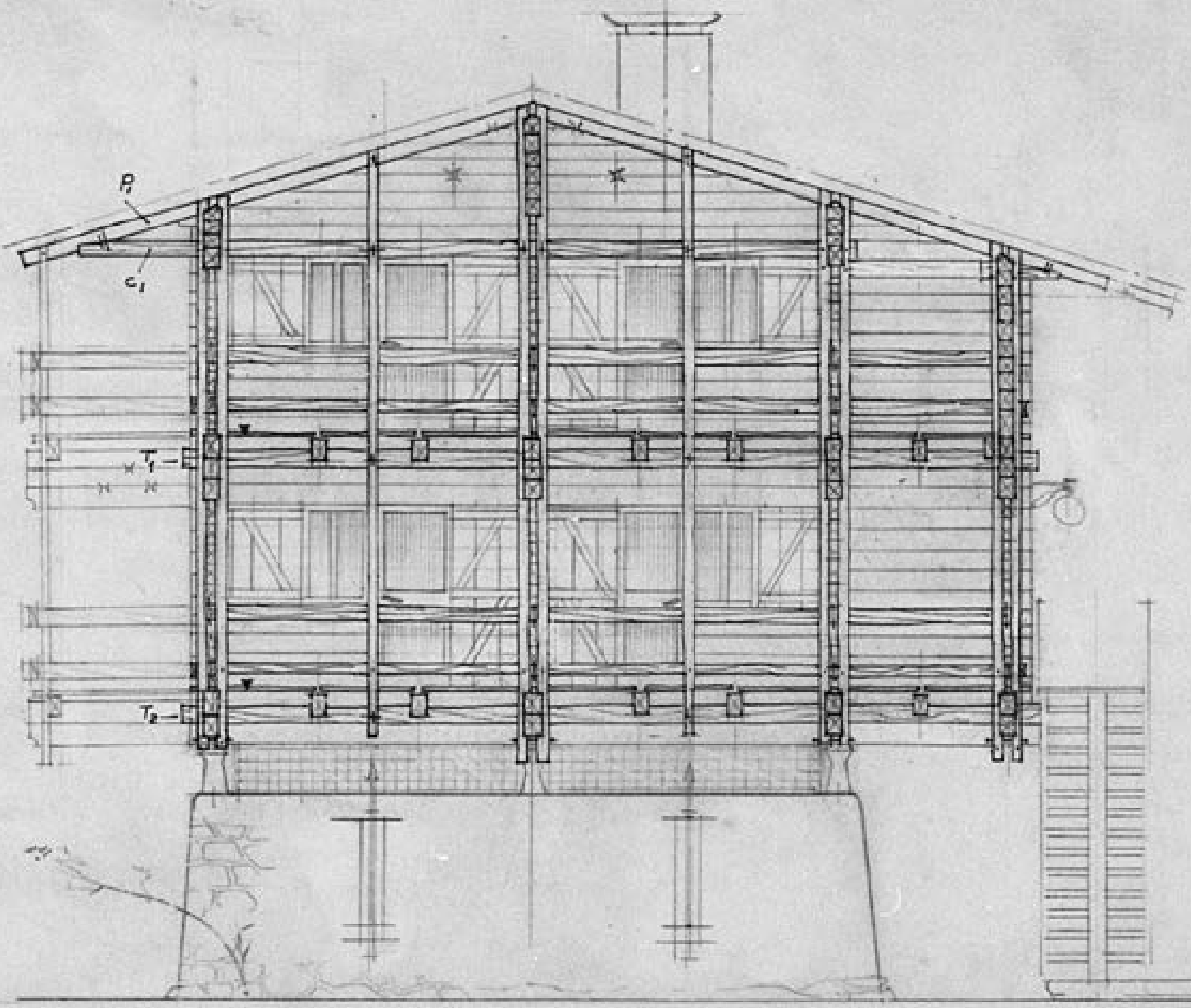

Fonte $\mathbf{I} \mathbf{C}_{1 \text { 1:50 }}$

(atholinente a owest)

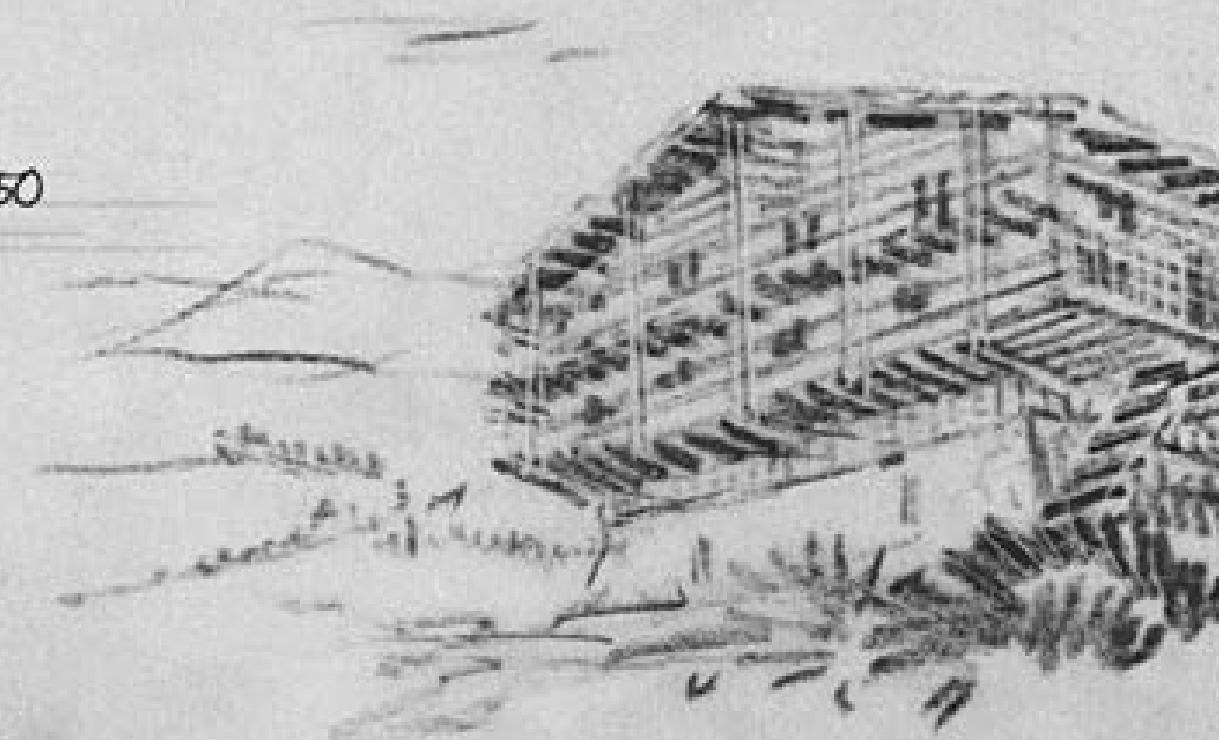




\title{
Manipolazioni metasemiche del patrimonio
}

\author{
Metasemic manipulations of heritage
}

\begin{abstract}
The construction of a renewed habitability of the contemporary Alpine space requires a profound critical revision of the ways of looking and of the cultures concerning the theme of re-use of the built heritage.

Over the last few decades, a sort of crystallization of imaginaries, operational practices and development ideas has emerged around the two terms of re-use and heritage and their ways of interaction, which today is likely to be an obstacle to the construction of new development scenarios for the Alpine region. Trying to imagine new values and meanings of the concepts of reuse and heritage, however, requires the questioning of those patrimonialization cultures that have served as the ultimate framework for the project of the Alpine space.

The essay reconstructs those design processes that, starting from a renewed productive vision of the mountain, attempt today to overcome a hypostatized vision of the conventional cultural landscape produced by the patrimonialist paradigm, to embrace a transformative attitude of the heritage based on the materic character of the basic elements of the Alpine space.

In particular we want to underline how the contemporary design culture in the Alps is directed to the development of synthetic languages aimed at capturing the stratified and diachronic dimension of the built landscape through metasemic cognitive and interpretative practices.

It is an attitude that, against a background of the change in perspective brought about by climate change and environmental issues, allows the maximization of the opportunities and physical resources recovered in the place, perfectly in line with the aptitude for the continuous re-use of the Alpine civilizations of the past, which focuses on the awareness of participating in a constructive process of transformation of the long lasting Alpine territory.
\end{abstract}

\begin{abstract}
Antonio De Rossi
Architect, PhD, full professor of architectural and urban design at Politecnico di Torino and director of the research centre "Istituto di Architettura Montana» (IAM). Between 2005 and 2014 he was vice director of «Urban Center Metropolitano» of Turin. He's author of various architectural projects in the Alps. He published the work in two volumes $L a$ costruzione delle Alpi (Donzelli 2014 and 2016) that have won the "Rigoni Stern" prize and the "Acqui Storia» prize and the book Riabitare l'Italia. Le aree interne tra abbandoni e riconquiste (Donzelli, 2018).
\end{abstract}

\begin{abstract}
Roberto Dini
Architect and PhD at the Politecnico di Torino, where he work as researcher and teacher. He studies the recent transformations of the alpine landscape and territory in the research centre "Istituto di Architettura Montana" (IAM). His publications include Architetture del secondo Novecento in Valle d'Aosta (2018), Rifugi e Bivacchi. Gli imperdibili delle Alpi (2018), Architecture in the Alps. Heritage and design (2017), Architettura alpina contemporanea (2012).
\end{abstract}

Keywords

Alps, heritage, contemporary architecture, reuse, sustainability. 
La costruzione di una rinnovata e per molti versi inedita abitabilità dello spazio alpino contemporaneo richiede, crediamo, una profonda revisione critica dei modi di guardare e delle culture concernenti il tema del riuso del patrimonio. Nel corso degli ultimi decenni, intorno ai due termini del $r$ uso e del patrimonio e alle loro modalità di interazione, si è infatti venuta a determinare una sorta di cristallizzazione di immaginari, pratiche operative e idee di sviluppo che rischia oggi di essere d'impedimento rispetto la costruzione di una nuova abitabilità del territorio alpino. Provare a immaginare nuove valenze e significati dei concetti di riuso e patrimonio necessita però la messa in discussione di quelle culture della patrimonializzazione che hanno funzionato da quadro di riferimento ultimo per il progetto dello spazio alpino.

Perché le culture della patrimonializzazione - o per meglio dire il paradigma patrimonialista -, che hanno dominato le visioni sulle Alpi nella fase recente, rischiano di essere un ostacolo al dispiegamento di un progetto di (ri)costruzione dell'abitabilità delle montagne? Per tre ragioni. La prima è che concepiscono il tema del patrimonio essenzialmente in termini di conservazione e valorizzazione delle eredità ambientali e storiche presenti nel territorio; conservazione e valorizzazione finalizzate in primis alla reiterazione di un'idea novecentesca di sviluppo della montagna incentrata in fondo sul consumo turistico. La seconda è che tali modalità di conservazione e valorizzazione determinano automaticamente la selezione di alcuni oggetti e azioni progettuali, a fronte dell'immenso stock di materiali e potenziali significati e valenze inscritto nel palinsesto alpino. La terza è che le culture della patrimonializzazione coltivano un'idea ridotta del patrimonio, riconducibile essenzialmente a questioni figurative e di paesaggio culturale, omettendo il ruolo che la matericità dei palinsesti può giocare nella costruzione di nuove forme di economia e società.

Costruire una nuova abitabilità significa quindi destrutturare gli sguardi che si sono venuti a consolidare nell'ultima stagione storica delle Alpi, al fine di riaprire nuovamente la partita. A partire da un ribaltamento di visione necessario. «Quas- sù c'è nulla da consumare. G'è invece molto da produrre», ha affermato recentemente Gianluca D'Incà Levis, curatore del progetto Dolomiti Contemporanee. Parole che trovano eco in una riflessione di un quarto di secolo fa del grande etnografo alpino Bernard Crettaz: «A forza di manipolazioni incessanti, le Alpi, miniaturizzate ed aggraziate, si sono esaurite. Sulle loro macerie una nuova cultura è alla ricerca di se stessa nelle montagne urbanizzate. Essa non ha ancora nome, né identità propria».

Costruire una nuova abitabilità delle Alpi significa allora partire da una rinnovata visione produttiva della montagna, in linea d'altronde con la lunga durata del vissuto storico alpino dal Medioevo fino all'avvio della modernità otto-novecentesca. Le risorse naturali - acqua, aria, boschi - e i resti, ruderi, rovine delle precedenti ondate trasformative dello spazio alpino, da meri elementi figurativi ipostatizzati di un paesaggio culturale convenzionale come quello prodotto dal paradigma patrimonialista, riacquistano una loro specifica matericità che può essere decisiva per la costruzione di una nuova civilizzazione delle montagne.

Con l'entrata nella modernità, ogni stagione storica ha sviluppato, nell'incontro tra montagne e città, un proprio specifico paradigma fatto di sguardi, concettualizzazioni, immaginari, pratiche nei confronti dei materiali costitutivi l'universo alpino. Modernità che agisce sulle Alpi per azioni certo metamorfiche - si pensi all'immane opera di infrastrutturazione per linee ferroviarie, dighe e centrali idroelettriche, sistemazioni idrogeologiche-, ma anche e soprattutto per manipolazioni metasemiche, operando continue mutazioni e ricombinazioni di significato sulla materia patrimoniale data, e spostando confini di senso e modalità relazionali tra gli oggetti.

Alcune esemplificazioni. La fase inaugurale della seconda metà del Settecento è stata fondamentale per conferire valenze, al contempo scientifiche ed estetiche, a spazi e fenomeni - ghiacciai, rocce, laghi - che fino a quel momento erano considerati alieni, determinando la nascita del paesaggio montano moderno. Il successivo paradigma del 
pittoresco alpino, tra l'inizio dell'Ottocento e il primo conflitto mondiale, costruisce attraverso la messa a punto del dispositivo estetico del contrasto complementare un'idea ricompositiva di paesaggio fondata sulla compresenza di elementi naturali, storici, tecnologici. Tutto l'Ottocento è d'altronde segnato da profonde metasemie: si pensi alla figura di Johannes Badrutt, artefice di Sankt Moritz e del turismo invernale, che attraverso un vertiginoso mutamento di senso trasforma la neve da fatto ostativo a elemento ludico e strumento di gioco. Ancora, l'ulteriore stagione del modernismo alpino, tra gli anni Venti e Settanta del Novecento, inventa attraverso il dispositivo del sublime tecnologico e la tecnicizzazione degli spazi dell'alta quota una nuova e inedita dimensione primigenia, ancestrale, assoluta della Natura.

Il punto di avvio del paradigma patrimonialista può essere ricercato nel celebre «discours de Vallouise», pronunciato dal presidente francese Valéry Giscard d'Estaing il 23 agosto 1977 durante una visita al Parc national des Écrins, che segna culturalmente e simbolicamente un vero e proprio crocevia nella storia recente delle Alpi. Chiude la stagione del modernismo novecentesco, e apre alla successiva - e tuttora in corso - fase della patrimonializzazione della montagna: critica alle forme di sviluppo e di turismo di tipo urbano e industriale, fine dell'esperienza delle megastazioni integrate invernali a favore di interventi di scala contenuta, difesa e conservazione dell'ambiente naturale, nuova centralità della storia e della cultura materiale alpina, inedita affermazione dell'importanza delle popolazioni montanare locali. Una visione che durante gli ultimi decenni dello scorso secolo ha avuto, come si è detto, un peso decisivo nella costruzione di nuovi immaginari e valori per il territorio alpino, e che ha trovato forte riscontro - dal punto di vista fisico - nei vasti fenomeni di valorizzazione e riuso del patrimonio architettonico e paesaggistico storico. Quasi improvvisamente, dopo la tabula rasa della modernità, insediamenti alpini storici, tracce e memorie delle antiche civilizzazioni montanare, conoscono un'inedita legittimazione e valore. Le parole d'ordine di questa nuova visione - autentico, tradizionale, tipico - riconfigurano in maniera pervasiva selezioni e gerarchie dei materiali costituenti le Alpi, determinando nuove immagini culturali e immaginari figurativi di paesaggio.

Un paradigma segnato al contempo da ambivalenze. Perché se è vero che la patrimonializzazione pone retoricamente al centro i temi della storia e della tradizione, dell'heritage e delle comunità locali, sovente restano immutate rispetto al Novecento le finalità e gli obiettivi, ossia l'idea che in fondo l'unica forma di sviluppo possibile per le Alpi sia quella turistica. L'opera - rilevante per le Alpi di un architetto come Michel Bezançon, che passa disinvoltamente dal progetto della futuristica stazione di La Plagne dei primi anni Sessanta al linguaggio néo-montagnard del villaggio reinventato di Valmorel abitato dai «touristes montagnards» della seconda metà degli anni Settanta, è emblematica di queste ambiguità, dove al mutamento radicale delle figurazioni corrisponde la continuità delle concettualizzazioni.

Ma la fase della patrimonializzazione vede anche l'emergere di un secondo fronte. I libri del geografo Werner Bätzing a partire dal 1984 dedicati alla regione alpina, i protocolli della Convenzione delle Alpi firmata il 7 novembre 1991 da tutti i paesi alpini europei, aprono a un inedito orizzonte, dove il riconoscimento del quasi infinito mosaico patrimoniale delle montagne - dalle risorse naturali (biodiversità, acqua, aria, geologia, ghiacciai, specie vegetali e animali, ecc.) fino a quelle storico-culturali (paesaggi, beni, tradizioni, lingue, alimentazione, ecc.) - viene a intrecciarsi con la questione, definita sempre più urgente, della costruzione di una nuova abitabilità del territorio alpino.

Il fenomeno della patrimonializzazione conoscerà declinazioni diverse nei paesi alpini di lingua latina e tedesca, e ciò determinerà l'affermarsi di nuove differenze e specificità regionali. Più forte l'accento sulla tradizione e la storia in quelli latini, maggiore l'attenzione all'innovazione tecnologica e alla sostenibilità ambientale in quelli di lingua tedesca.

Ma la fase della patrimonializzazione determinerà soprattutto l'emergere di una nuova produzione architettonica di qualità che, a partire dalla Cappella di Sogn Benedekt di Peter Zumthor del 1988, segna non solo il superamento della consueta e ipostatizzata dicotomia tra modernità e tradizione intorno cui si erano costruite le retoriche dell'architettura montana del Novecento, ma l'emergere di pratiche e atteggiamenti che consentono di guardare ai materiali patrimoniali del mondo alpino da altri, inaspettati, punti di vista. In un saggio del 1996 pubblicato sulla rivista elvetica «Faces», dal titolo Découvrir le monde des choses e dedicato alla nuova architettura dei Grigioni, il critico Martin Steinmann scriveva parole preziose circa la possibilità di costruire inedite forme di ricerca architettonica a partire da procedure denaturalizzanti e atti metasemici: «Ge mot "découvrir" est tombé plus d'une fois dans notre entretien: découvrir, par exemple, d'autres manières d'utiliser les matériaux, le bois, la pierre, la brique,... et en faire ressortir de nouveaux effets. Mais il ne s'agit pas de faire du nouveau pour faire du nouveau: il s'agit de trouver la face cachée des choses 
familières, cela dans l'intention de désautomatiser le terme est des formalistes russes - une connaissance qui, par la familiarité des choses, a cessé d'être connaissance».

Un sottile gioco in bilico tra spaesamento e costruzione di rinnovate appartenenze non privo di precedenti tra le Alpi, se si pensa ad esempio all'opera architettonica di Carlo Mollino, in cui le operazioni di smontaggio e rimontaggio, di ripresa e distorsione, di decontestualizzazione e di rimessa in cornice degli elementi storici e patrimoniali acquisiscono valore centrale, come nel caso emblematico del progetto per il Rascard Garelli a Champoluc nel 1963-65.

Naturalmente, non si tratta di un mero tema formale di ritrovamento e apertura di inediti significati degli oggetti patrimoniali. A richiedere una riconcettualizzazione dei materiali costituenti il palinsesto alpino è il profondo mutamento di prospettiva determinato - sulle Alpi per certi versi più che altrove - dal cambiamento climatico e dalle questioni ambientali. La recente tempesta Vaia, che ha colpito ampie aree delle Alpi centro-orientali italiane alla fine del mese di ottobre 2018 distruggendo completamente alcune decine di migliaia di ettari di foreste, pone questioni oggi centrali che hanno valenza metonimica: ripristinare i boschi preesistenti, dominati dalla monocoltura dell'abete rosso, riedificando quindi il precedente paesaggio culturale, o viceversa progettare uno spazio ambientale differente, nell'incrocio tra mutamenti climatici e nuove visioni dello spazio montano?

Questi temi cambiano radicalmente lo sfondo concettuale e operativo delle culture progettuali di riuso del patrimonio. Non si tratta solamente della fine di quell'idea di crescita che ha accompagnato - con tutte le sue contraddizioni e nuove diseguaglianze - la lunga fase del neoliberismo a cavallo del passaggio di secolo. Se lo sviluppo sostenibile è il solo orizzonte perseguibile, a modificarsi è innanzitutto la concezione stessa di modificazione dell'ambiente, con il prevalere di una visione che mette al centro le figure del riuso e del riciclo come uniche pratiche costruttive possibili per il futuro. La conservazione delle risorse ambientali e del suolo, l'idea che le trasformazioni debbano avvenire prevalentemente all'interno delle linee di inviluppo di quanto è già stato modificato e costruito, l'attenzione per le questioni energetiche e per l'innovazione tecnologica, destrutturano l'idea di riuso nata durante la stagione patrimonialista, le sue istanze e principi valoriali. Quello cui si assiste pare dunque configurarsi come un vero e proprio cambio di paradigma nello statuto disciplinare dell'architettura e dei saperi concernenti il territorio, in cui i temi del riuso, del riciclo, della riscrittura e della manipolazione dell'esistente disegnano il campo della dialettica tra ambiente e azioni antropiche.

Con uno spostamento significativo. Perché se il principio di autorità che guida la determinazione dei confini patrimoniali non è più costituito meramente dal valore e dalla possibile valorizzazione del bene, ecco allora che si determina un immediato ampliamento a dismisura dei materiali ritenuti operabili. Non più solamente gli spazi e manufatti ritenuti di valore storico e ambientale, ma anche l'edificato recente, gli oggetti della colonizzazione turistica della montagna - tutti manufatti in stato di sottoutilizzo e talvolta di abbandono -, fino alle sistemazioni ambientali e insediative operate storicamente nello spazio alpino.

Non si tratta però solamente di un tema quantitativo di disponibilità patrimoniale: il cambiamento climatico e le relative problematiche idrogeologiche, la necessità di un'operazione di ripensamento tout court delle strutturazioni insediative alpine dopo i disequilibri ingenerati dalle differenti fasi di modernizzazione, e soprattutto le recenti dinamiche di reinsediamento in atto con le loro inedite istanze di abitabilità del territorio (rigenerazione a base culturale, nuove forme di agricoltura e turismo, processi di innovazione sociale, ripensamento del welfare, trasferimento tecnologico) rendono le Alpi un laboratorio ideale per sperimentare nuove pratiche architettoniche di riuso.

Sotto questo profilo la cultura architettonica sulle Alpi ha mostrato negli ultimi anni una particolare sensibilità ai temi del ripensamento e riutilizzo del patrimonio secondo un'accezione non ideologicamente conservativa e di mera valorizzazione, e che alla luce delle nuove esigenze oltrepassa la dimensione patrimonializzante per abbracciare invece un approccio basato sulla sua rifunzionalizzazione che è al contempo operazione di risignificazione a tutto tondo. Una sorta di «estensione del dominio del patrimonio» - per citare Nathalie Heinich - che tra le Alpi ha progressivamente spostato il punto di vista da una propensione inventariale ad una prospettiva progettuale, e in cui la riflessione sulla matericità delle cose assume valenza prioritaria.

In quest'ottica, l'architettura contemporanea sulle Alpi sembra aver sviluppato originali percorsi di ricerca progettuale su questo tema, superando sia un approccio finalizzato al perseguimento della dimensione valoriale contenuta nella preesistenza, sia la retorica della contrapposizione antico-nuovo che ha guidato un'ingente parte degli interventi sull'esistente nei decenni recenti, quasi una riproposizione - si pensi alla dialettica tra materiali come la pietra e il corten - dell'ottocente- 
sco dispositivo del contrasto complementare. Una nuova attitudine in grado dunque di intrecciare azioni di tutela critica con consapevoli pratiche di manipolazione degli oggetti.

Ma il dato più rilevante è forse ancora un altro. Attraverso tali operazioni manipolatorie, sovente assai prossime alle procedure della ricerca artistica contemporanea, questi progetti architettonici ridefiniscono i confini del campo patrimoniale, e al contempo disvelano nuovi significati e valenze contenute nella matericità dei palinsesti alpini, aprendo nuove possibili interpretazioni e declinazioni del rapporto con l'ambiente, la storia, la morfologia e la fisicità delle cose della montagna. È un'opera di manipolazione che procede per straniamenti e disvelamenti finalizzata a togliere incrostazioni culturali agli sguardi con cui osserviamo il mondo delle cose, e che proprio per questo agisce per piccoli gesti sovente artigianali, capaci di mettere in valore le patine materiche dei manufatti. Manipolazioni e spaesamenti che permettono alla pietra e al legno di ritornare a essere usati per la loro essenza e potenzialità tettonica e costruttiva, per la loro geometria e possibile apparecchiatura e tessitura. Insediamenti alpini storici che strappati dalla stratificazione delle connotazioni simboliche e culturali convenzionali vengono reimmessi nel flusso del tempo a partire dalla loro consistenza di atti costruttivi.

Una riconcettualizzazione del mondo delle cose che passa da una necessaria rifisicizzazione della realtà materica.

In particolare si vuole sottolineare come la cultura progettuale contemporanea nelle Alpi dai caratteri maggiormente sperimentali sia indirizzata allo sviluppo di architetture altre che ricompongono in un'unica figurazione - ma senza celarne le discontinuità e le rotture - la dimensione stratificata e diacronica degli oggetti costruiti. Non è quindi un tema di restauro tradizionale, anche se il restauro rimane in alcuni casi una possibile strategia degli interventi. Semmai si tratta di leggere i modi con cui il progetto consente di operare una sintesi intenzionalmente non risolta e volutamente tensionale - come nel caso delle rovine, indagate da Georg Simmel in un bellissimo saggio nel 1911 tra dimensione storica e dimensione contemporanea, con esiti che trascendono le componenti di partenza: uno + uno $=$ tre.

Ciò che interessa è dunque l'interazione tra il vecchio ed il nuovo nella misura in cui questa genera un'architettura terza che li contiene entrambi - sia nel rispetto della profondità storica e della lunga durata dei processi insediativi alpini, sia nel soddisfacimento delle attuali esigenze abitative e delle logiche di sviluppo locale, per le quali come si è visto il patrimonio esistente costituisce un punto di riferimento essenziale -, riuscendo al contempo a oltrepassare, grazie a pratiche interpretative e metasemiche, queste due dimensioni.

Lavorare a favore dell'intreccio, della compenetrazione tra presente e passato, tra costruito e natura, tra spazi del lavoro e dell'abitare, significa però necessariamente mutare il paradigma che sta alla base del progetto di architettura e di territorio. Significa depotenziare la centralità dell'atto costruttivo e trasformativo come telos del fare progettuale, per privilegiarne la valenza euristica e cognitiva. In questo modo il progetto non è più semplicemente ciò che il progettista aggiunge, ma l'assetto complessivo di un luogo o di un manufatto, esito fisico dell'insieme degli elementi che sono stati rinvenuti e successivamente confermati o modificati, oppure introdotti ex novo, a partire dalla valenza strategica dell'atto di risignificazione.

Un atteggiamento di massimizzazione delle opportunità e risorse fisiche ritrovate nel luogo, perfettamente in linea con l'attitudine al continuo riuso delle civiltà alpine del passato, che mette al centro la consapevolezza di partecipare a un processo costruttivo di trasformazione del territorio alpino di lunga durata. 


\section{tumulazione}

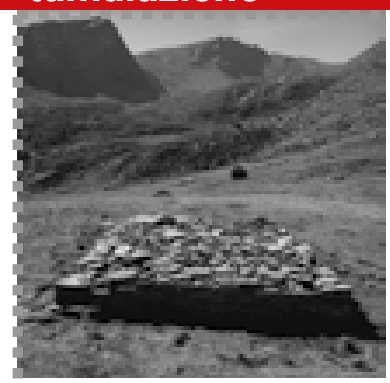

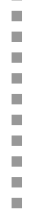

\section{restituzione morfologica}

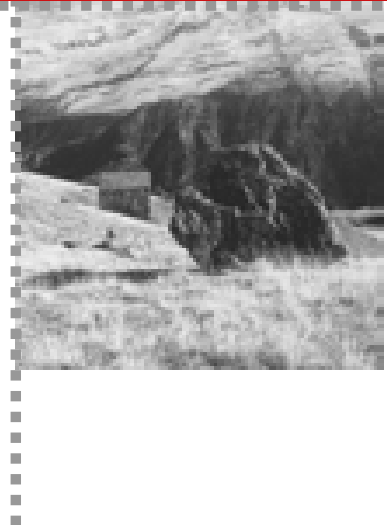

Grado di riprocessamento della preesistenza

\section{sottolineatura, messa in cornice, amplificazione}

reinterpretazione semantica e materica
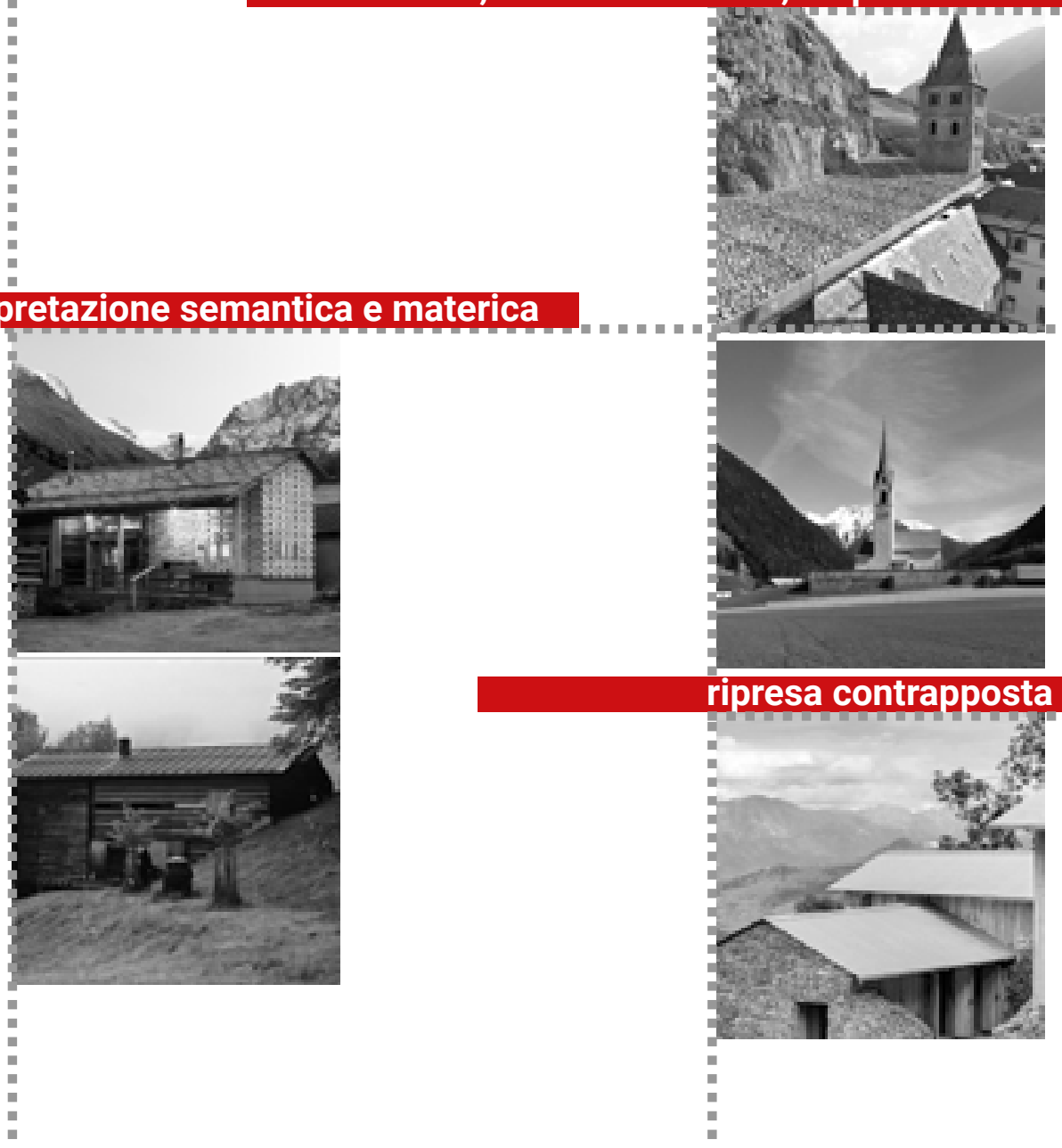


\section{I progetti}

Una lettura comparata di alcune realizzazioni recenti sulle Alpi mette in luce differenti famiglie di azioni progettuali sull'esistente con gradienti e sfumature che sembrano muoversi su due assi principali.

Lungo una direzione abbiamo il grado di manipolazione della preesistenza stessa che si muove da una continuità molto aderente al manufatto originale fino ad interventi di marcata trasformazione.

Nell'altra direzione sembra invece esserci il $\mathrm{gra}$ do di riprocessamento dell'architettura esistente che si colloca tra i limiti di un metamorfismo in cui troviamo una mutazione della fisicità degli oggetti ed una metonimia che presenta vere e proprie alterazioni di significato.

Una prima famiglia di progetti ricerca una dialettica incrociata basata sulla continuità con la preesistenza attraverso una reinterpretazione semantica o materica di ciò che già esiste.
È il caso ad esempio del progetto per casa Gugalun nei Grigioni di Peter Zumthor dove il nuovo ampliamento si caratterizza come un'estrusione del volume preesistente e dove il gioco astratto dell'involucro in lamelle di legno è sapientemente utilizzato per riprendere la disposizione tipica del blockbau tradizionale. Un progetto recente che presenta forti analogie è quello dell'ampliamento della Grange Satarma in Vallese di Galletti-Matter dove di nuovo la reinterpretazione in chiave stilistica di un sistema costruttivo diventa la texture di facciata.

Altri progetti sviluppano invece una sorta di ripresa contrapposta in cui è compresente l'idea di un completamento del volume originario che però viene realizzato secondo un principio di marcata rottura e contrasto costruttivo, materico o geometrico con la preesistenza.

Significative sono alcune esperienze recenti nelle alpi piemontesi come gli interventi dell'architetto

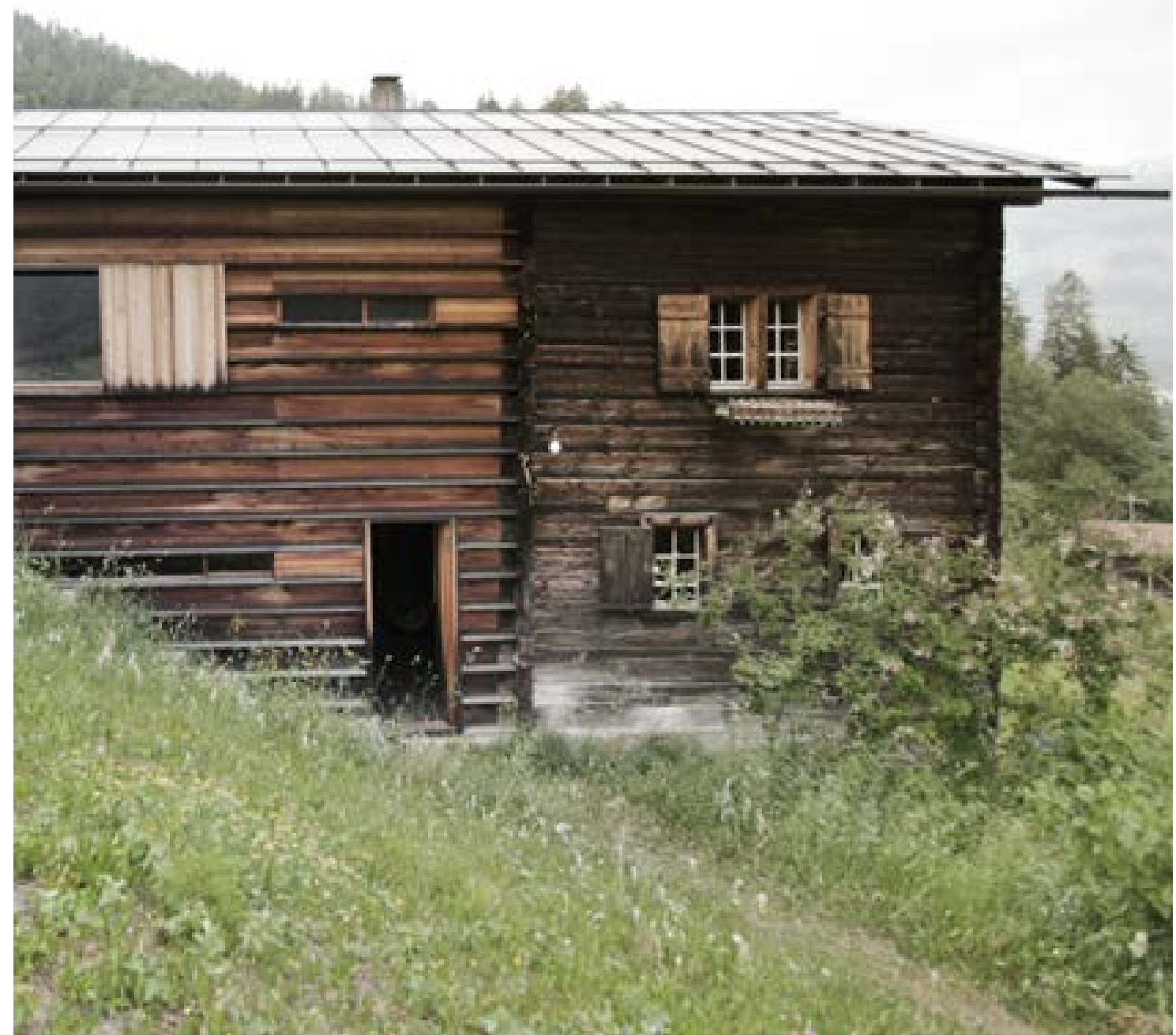



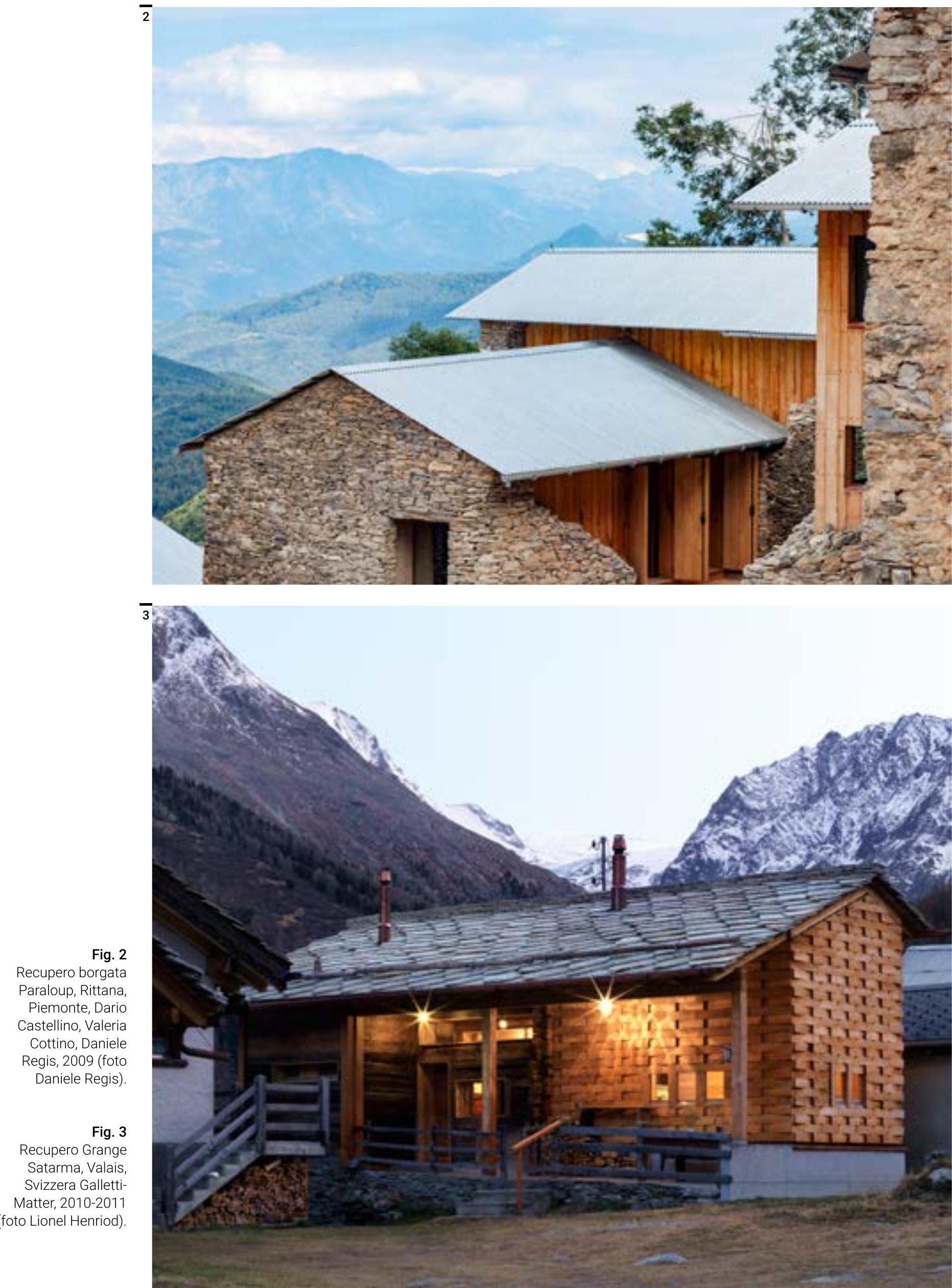
Dario Castellino come ad esempio il recupero della borgata Paraloup che sviluppa il tema del "riabitare la rovina". Si tratta di una modalità colta di reinterpretare la preesistenza che ne riprende idealmente le volumetrie e le proporzioni attraverso un completamento volto ad esplicitare la differenza tra le parti nuove e quelle originarie.

A ciò si accompagna l'utilizzo di materiali poveri e tecniche costruttive minimali in linea con le strut- ture tipiche del mondo rurale fatte di muri in pietra a secco, strutture di tamponamento e di copertura in legno e lastre di lamiera.

$\mathrm{Si}$ tratta dunque di interventi dal linguaggio contemporaneo che instaurano una forte relazione con la cultura costruttiva storica locale, reinterpretandone in chiave attuale tecniche e materiali. Un aspetto di estrema aderenza alla preesistenza è invece quello perseguito attraverso quei lavori

Fig. 4 Restauro Türalihuus,

Valendas,

Grigioni, Capaul \& Blumenthal, 20072014 (foto Ralph Feiner)

Fig. 5 Cinema Sil Plaz, Ilanz, Grigioni, Capaul \&
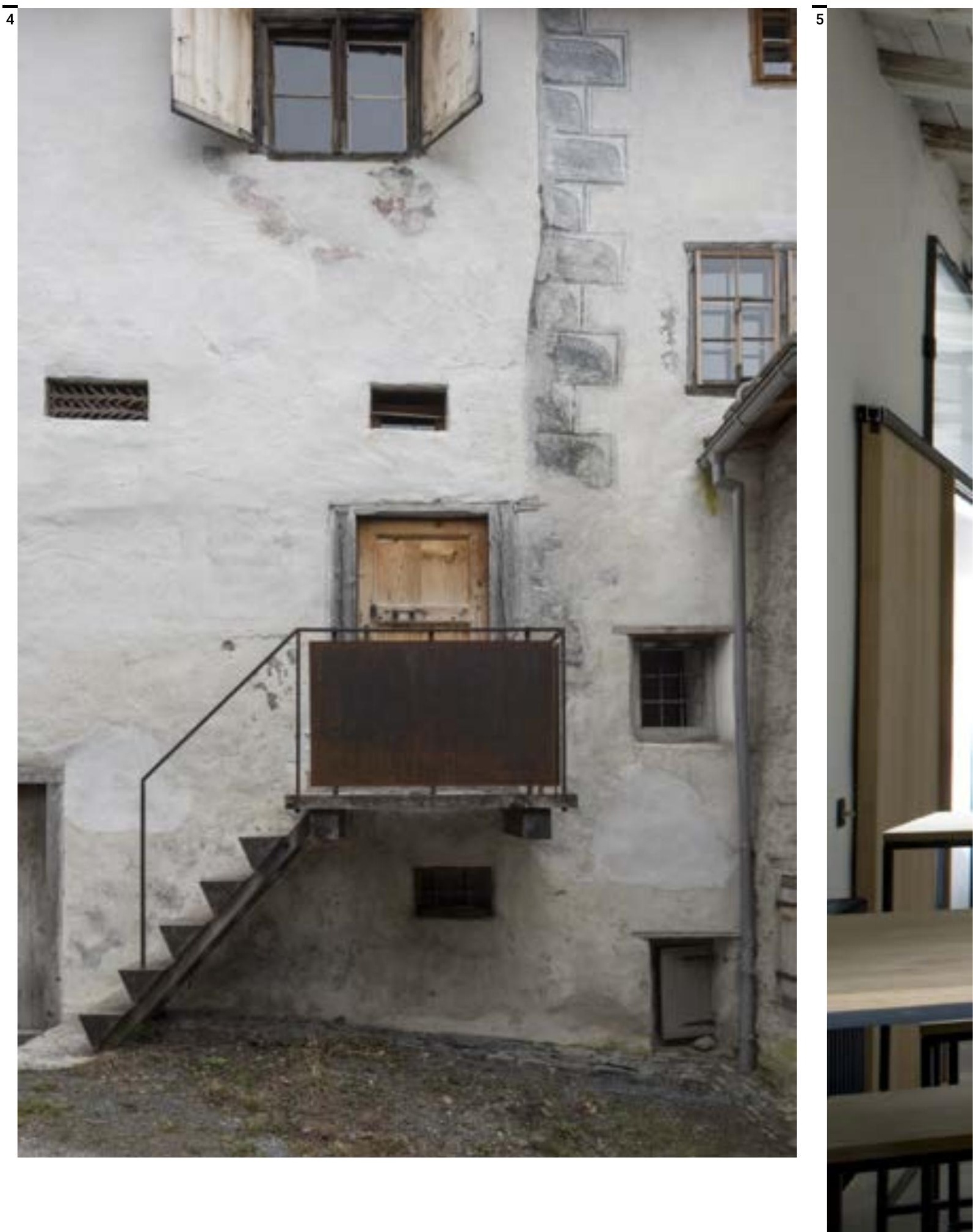
volti a congelare uno stato di fatto attraverso una sorta di fissazione estraniante. In questi casi, la valorizzazione radicale delle sovrapposizioni diacroniche, spaziali e materiche dell'edificio è fatta con la conservazione e la messa in tensione di compresenze contrastanti e spaesanti. Pensiamo ad esempio al progetto di restauro conservativo per la Türalihuus di Valendas fatto da Capaul \& Blumenthal che, nella volontà di preservare le diffe- renti fasi che hanno caratterizzato la storia dell'edificio, hanno preferito evitare qualsiasi sorta di ricostruzione filologica di una fase prevalente scegliendo una strada volta a valorizzare la sovrapposizione delle tracce del passato. Per queste ragioni si è operato sul frammento, sulle tracce, sulla riparazione degli elementi danneggiati, sulla conservazione degli inestetismi come l'annerimento dei muri provocato dal fumo o i segni dell'usu-

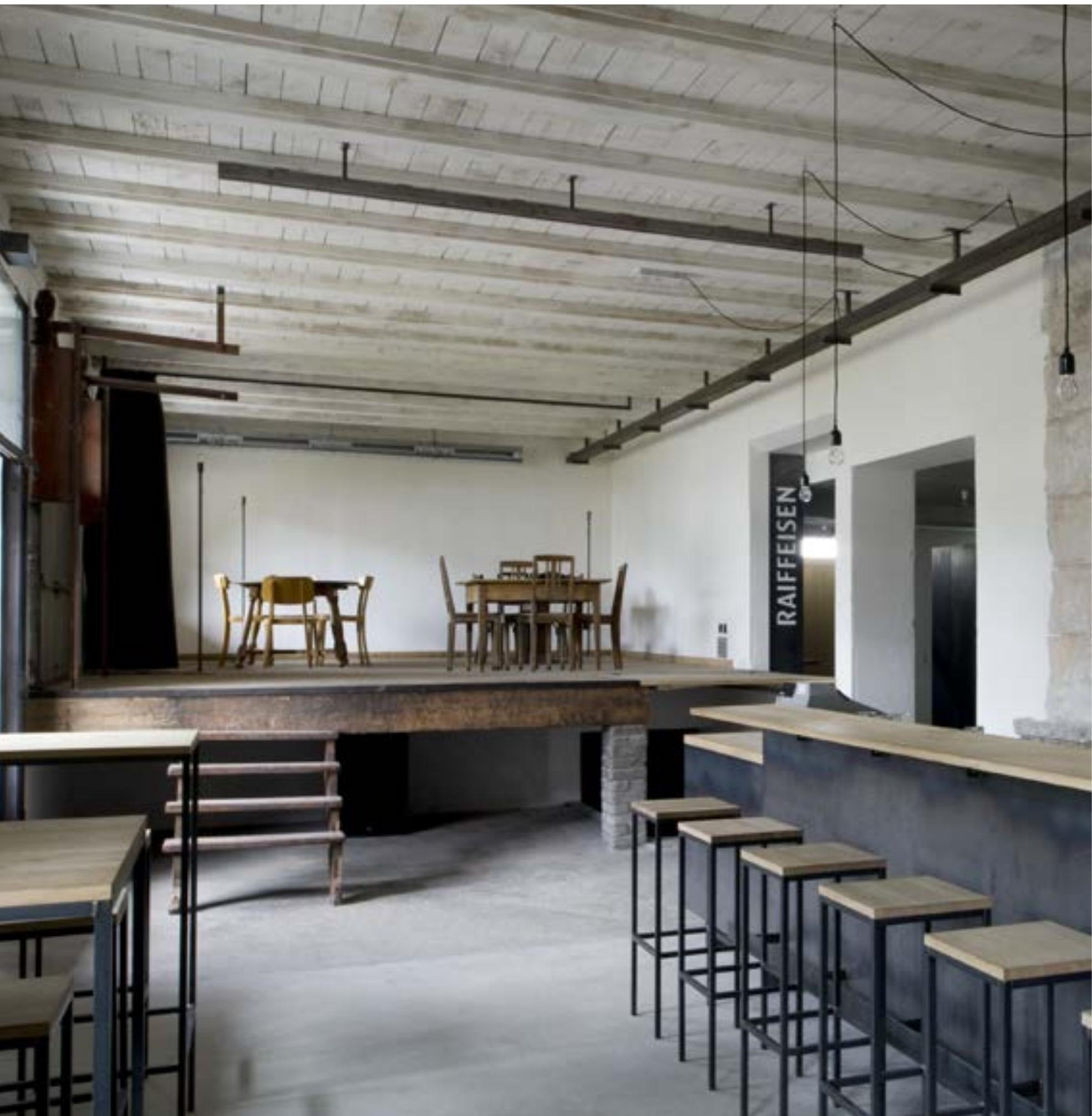


ra, dando vita ad una sorta di "brutalismo della conservazione".

Un'altra tipologia di intervento è volta invece al mantenimento della matrice storica e alla sua messa in tensione attraverso operazioni di innesto perturbante che, attraverso l'inserimento di elementi contemporanei, consentono lo svelamento di nuovi significati, nuove spazialità, nuove soluzioni architettoniche, costruttive e materiche.

Pensiamo ad esempio al significativo progetto per il recupero di un'antica rimessa agricola fatto dagli architetti Marques \& Zurkirchen a Bergün nei

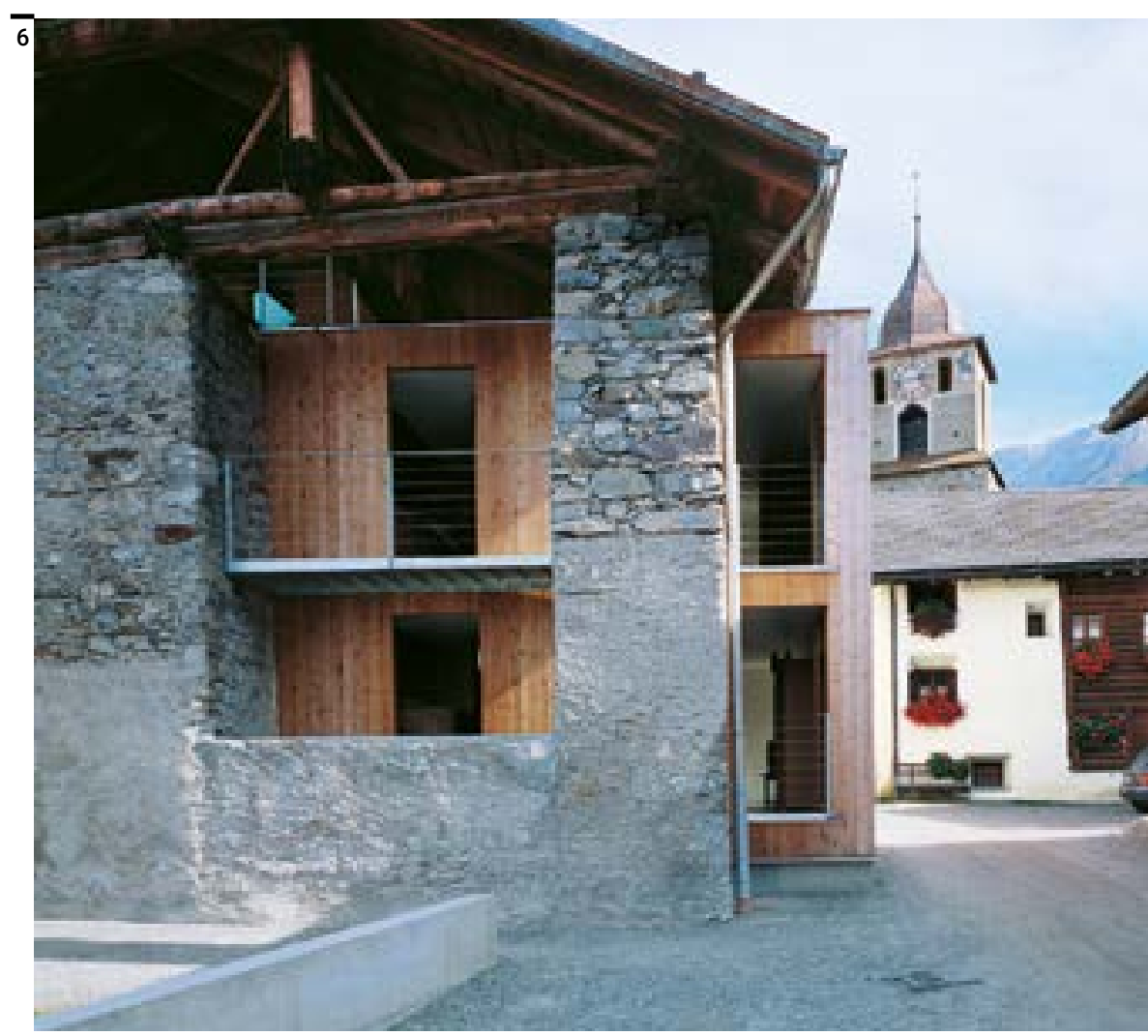

Fig. 6

Recupero a Bergün, Grigioni, Marques \& Zurkirchen, 1996 (foto Ignacio Martinez)

Fig. 7

Chesa Not, Ruch \& Partner Architekten AG, 2004, Tschlin (foto Filippo Simonetti)

Figg. 8-9 Recupero del Forte di Fortezza, Markus Scherer con Walter Dietl, 2009 (foto René Riller).

Fig. 10 Recupero del Forte di Fortezza, Markus Scherer con Walter Dietl, 2009 (foto Alessandra Chemollo) $\overline{8}$

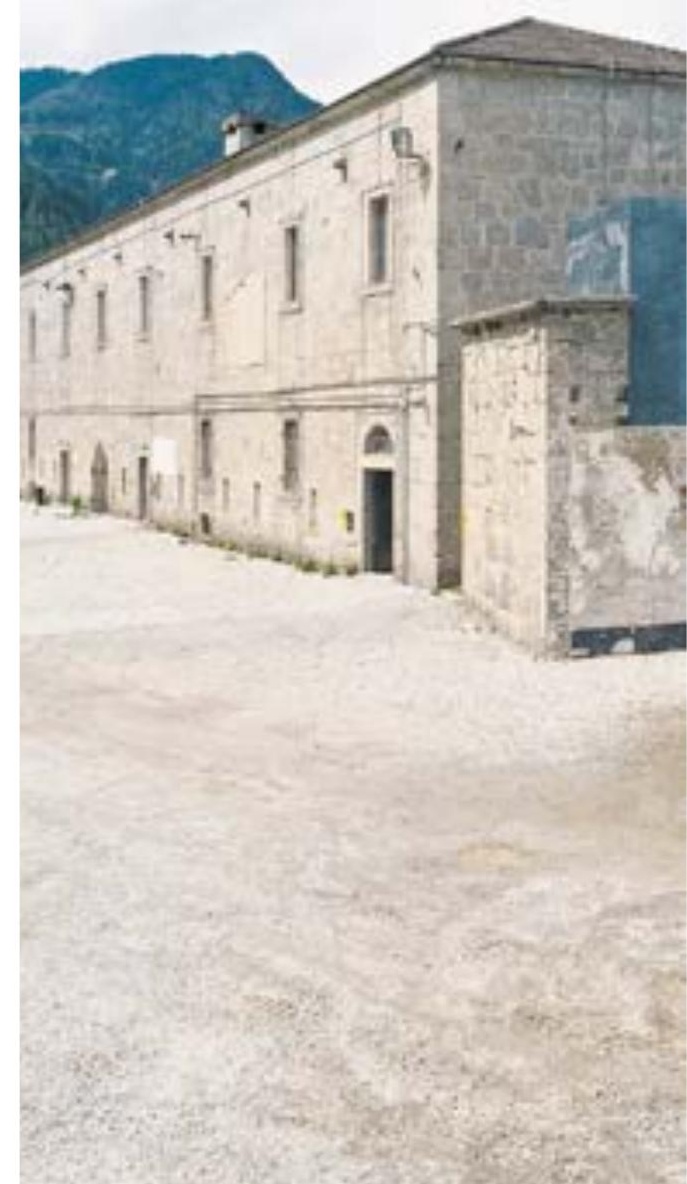


Grigioni dove un nuovo volume indipendente con struttura in legno e metallo viene inserito all'interno dell'involucro originario dell'edificio consentendone una rinnovata abitabilità.

Di notevole interesse sono da questo punto di vista i progetti di recupero di "Chese" engadinesi re- alizzati dall'architetto Ruch nei Grigioni: volumi sospesi, controsoffittature avvolgenti, nuove pareti divisorie e serramenti vengono inseriti in contrapposizione agli spazi originari del vecchio edificio. Anche nel progetto di riqualificazione del Forte di Fortezza elaborato da Markus Scherer vi è una
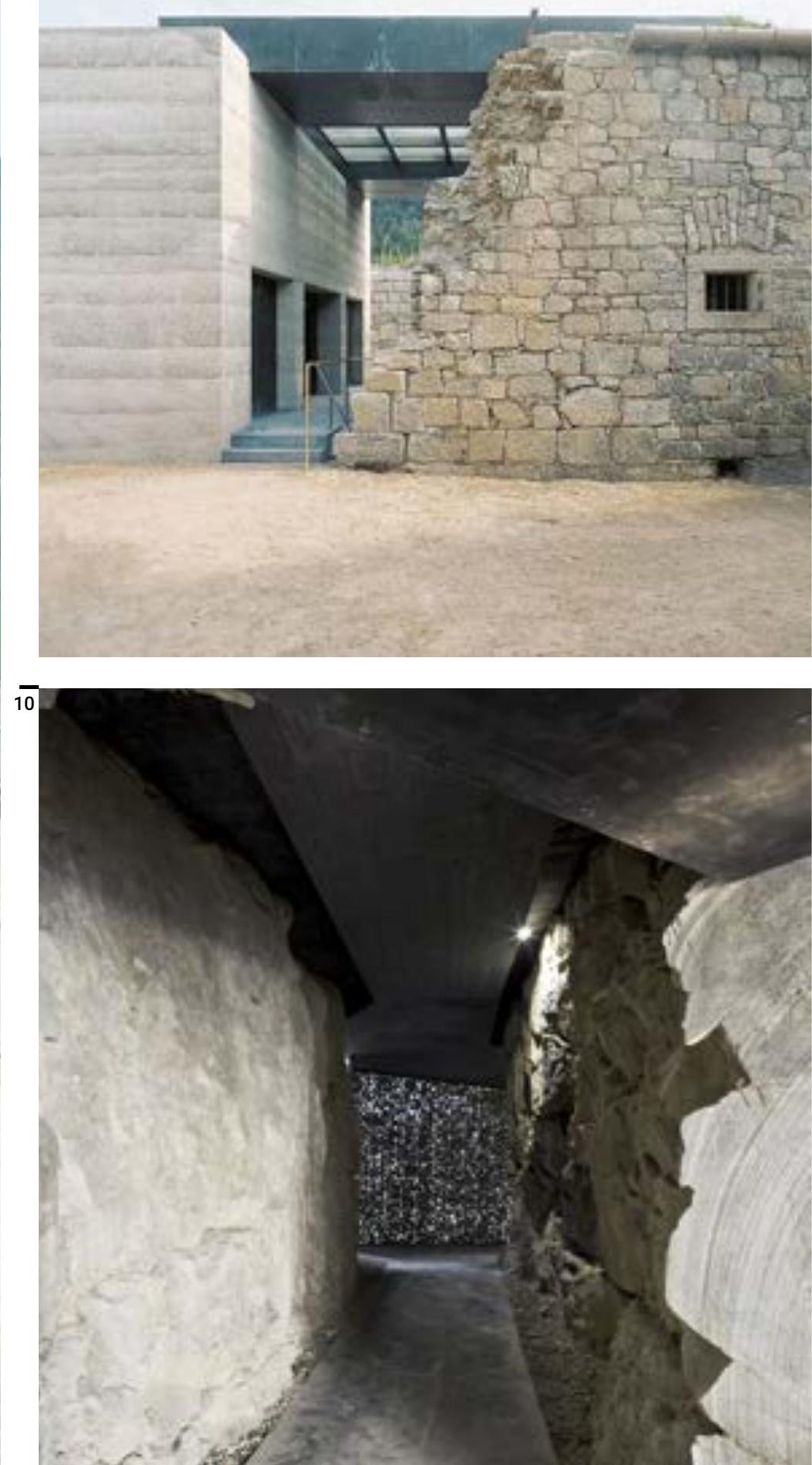
forte compenetrazione di elementi contemporanei in quelli che erano gli spazi della costruzione storica. Accanto alla conservazione delle antiche cortine murarie che vengono preservate intatte vi è infatti la realizzazione di nuovi volumi e strutture che creano spazi, percorsi, nodi e attraversamenti in grado di svelare e generare una fruibilità nuova, originale e inattesa del bene.

Una ulteriore famiglia di interventi mette in atto delle azioni di sottolineatura-messa in cornice-amplificazione che, a scale differenti, creano una sorta di punteggiatura architettonica volta alla messa in valore di ciò che già esiste. Questo approccio si caratterizza in molti casi per la conservazione radicale della materia storica attraverso il solo ripristino della fruibilità della preesistenza.

Pensiamo alle opere di riqualificazione in chiave turistica e museale di alcuni castelli in Sudtirolo come ad esempio il recupero di Schloss Tirol di Walter Angonese o di Firmiano di Werner Tscholl dove scale, passerelle pedonali, piccoli box, volumi di servizio, realizzati prevalentemente attraver- so superfici astratte e metalliche, come l'acciaio corten, consentono di rendere fruibile e accessibile a fini museali le rovine e gli spazi degli antichi manieri.

Si tratta di interventi che intendono rispettare le caratteristiche spaziali e costruttive del manufatto attraverso soluzioni non impattanti, reversibili e non mimetiche al fine di non alterare la storicità del bene, fatti con elementi ed inserti essenziali che hanno un linguaggio unitario e minimale proprio per non intaccarne l'aspetto architettonico consolidato.

In altri progetti, si pensi agli ampliamenti dei due cimiteri di Lutago e di San Sigismondo in Sudtirolo di EM2 Architekten, le azioni di sottolineatura fatte con le nuove cortine murarie diventano delle vere e proprie opere di arte paesaggistica che rimettono in riverberazione le preesistenze storiche della chiesa e del villaggio con l'ambiente naturale circostante.

Nel progetto per la copertura degli scavi archeologici all'abbazia di Saint-Maurice di Savioz Fabriz-
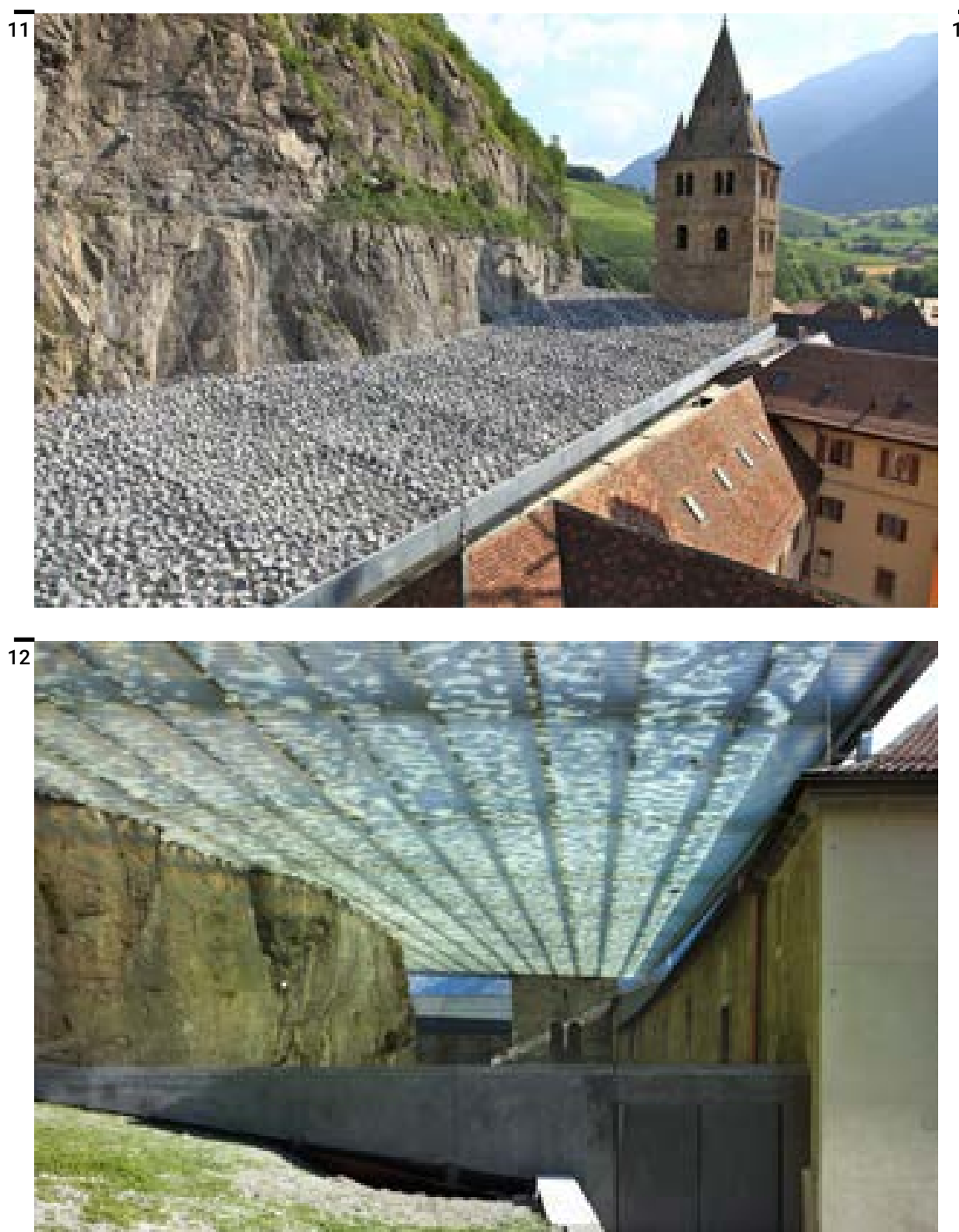

$\overline{13}$

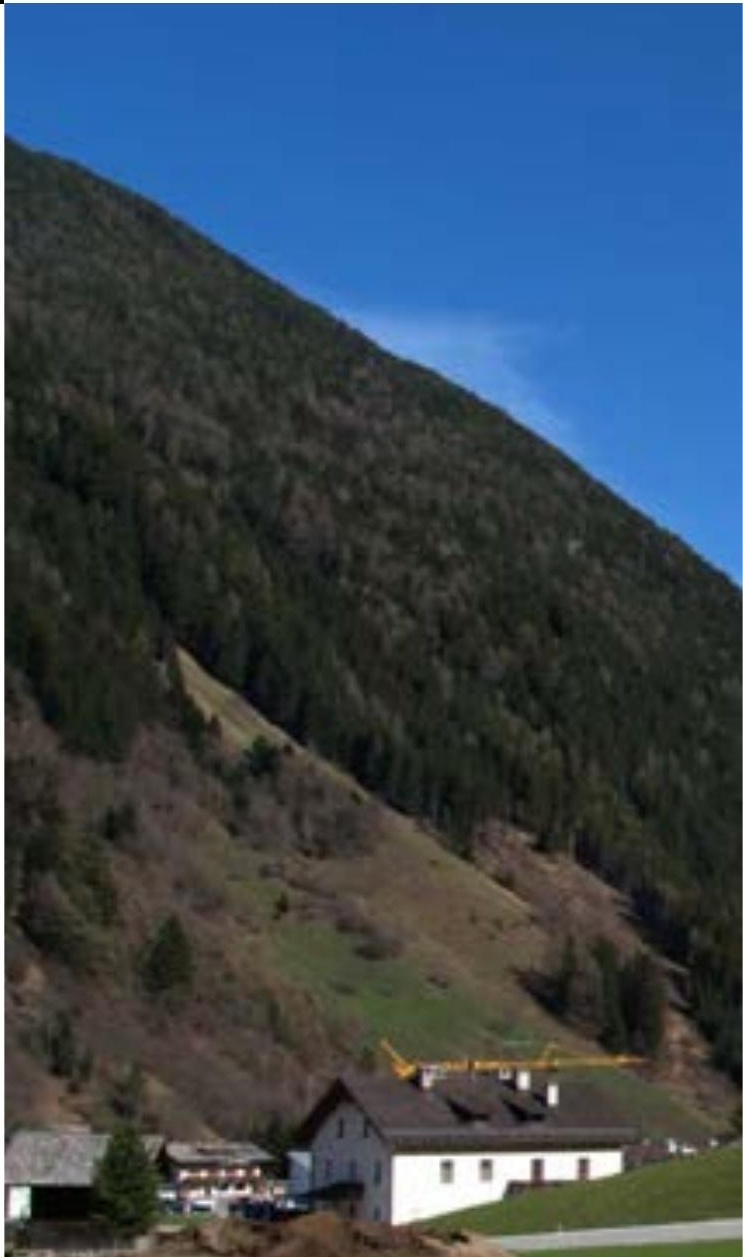


Figg. 11-12 Copertura scavi archeologici all'Abbazia di SaintMaurice, Sion, Savioz Fabrizzi, 2010 (foto Thomas Jantscher).

Fig. 13 Ampliamento cimitero di Lutago, Alto Adige, EM2 Architekten, 20022004 (foto EM2 Architekten).

Fig. 14

Recupero Castel Firmiano - Messner Mountain Museum, il cinema, Alto Adige, Werner Tscholl, 2009-2016 (foto Eleonora Gabbarini).
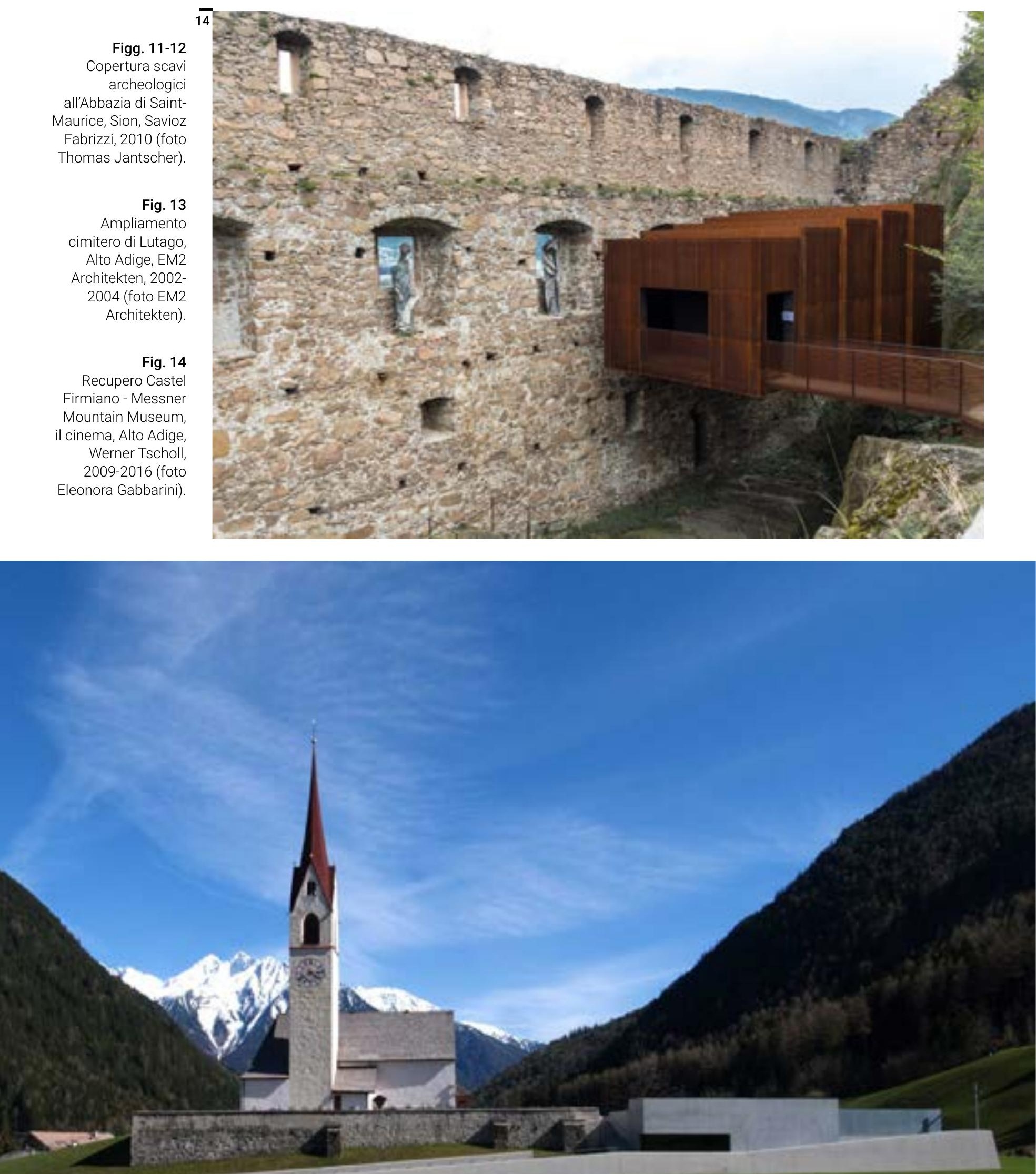
zi a Sion in Vallese, la struttura metallica di protezione dell'area sottostante crea invece un suolo sospeso che reinventa un nuovo spazio di relazione tra le diverse parti del complesso ecclesiastico, amplificando la relazionalità tra campanile, edificio a corte e falesia rocciosa.

Un'ultima serie di interventi mette infine in atto una trasformazione estremamente radicale del manufatto di partenza che, oltre a mutarne aspetto e modalità di fruizione, opera delle vere e proprie operazioni metonimiche di carattere concettuale ed artistico.

Pensiamo ad esempio alla Gelbe Haus a Flims dove Valerio Olgiati interviene secondo un principio di metonimia metamorfica, svuotando l'interno dell'edificio, modificandone parzialmente il sistema costruttivo attraverso l'inserimento di un pilastro sfalsato rispetto alla maglia strutturale originaria, e operando una sorta di scorticazione dell'involucro esterno che viene poi ricoperta da uno strato di calce bianca. L'edificio cambia volto senza tuttavia perdere la configurazione originaria ma acquisendo invece significati e spazialità inedite.
Di notevole interesse sono anche quegli interventi di ristrutturazione radicale progettati dagli architetti vallesani Savioz Fabrizzi, come la casa Savioz a La Giète-Délé, dove la matrice dell'edificio in pietra originario viene completata attraverso una sorta di consolidamento brutalista fatto con il cemento armato che solidifica la preesistenza e poi con la successiva tinteggiatura coprente che annulla le caratteristiche cromatiche dei diversi materiali operando una sorta di omogeneizzazione astraente dell'involucro.

Progetti come la casa Roduit a Chamoson, sempre di Savioz Fabrizzi, presentano una forte componente di reinvenzione tettonica volta a ridefinire i canonici rapporti tra tecnica e forma degli edifici preesistenti per svelare nuovi possibili modi di intendere l'identità morfo-tipologica del manufatto. Di notevole interesse l'approccio di Martino Pedrozzi nei suoi lavori di smontaggio e ricomposizione di alcune malghe in Canton Ticino attraverso un intervento di definitiva tumulazione dei ruderi o semplicemente con la "smussatura" di alcuni manufatti in abbandono nell'intento di tra-

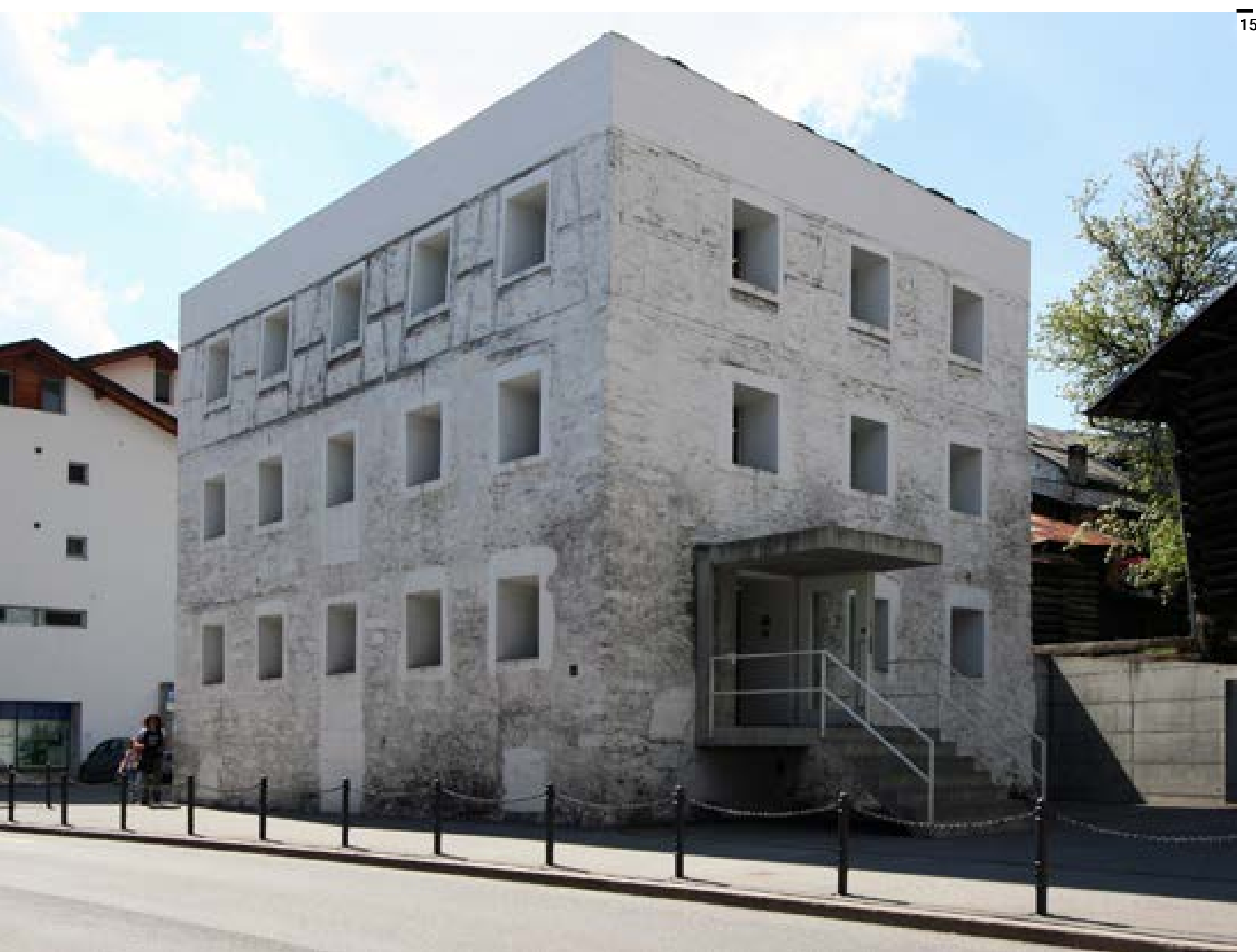




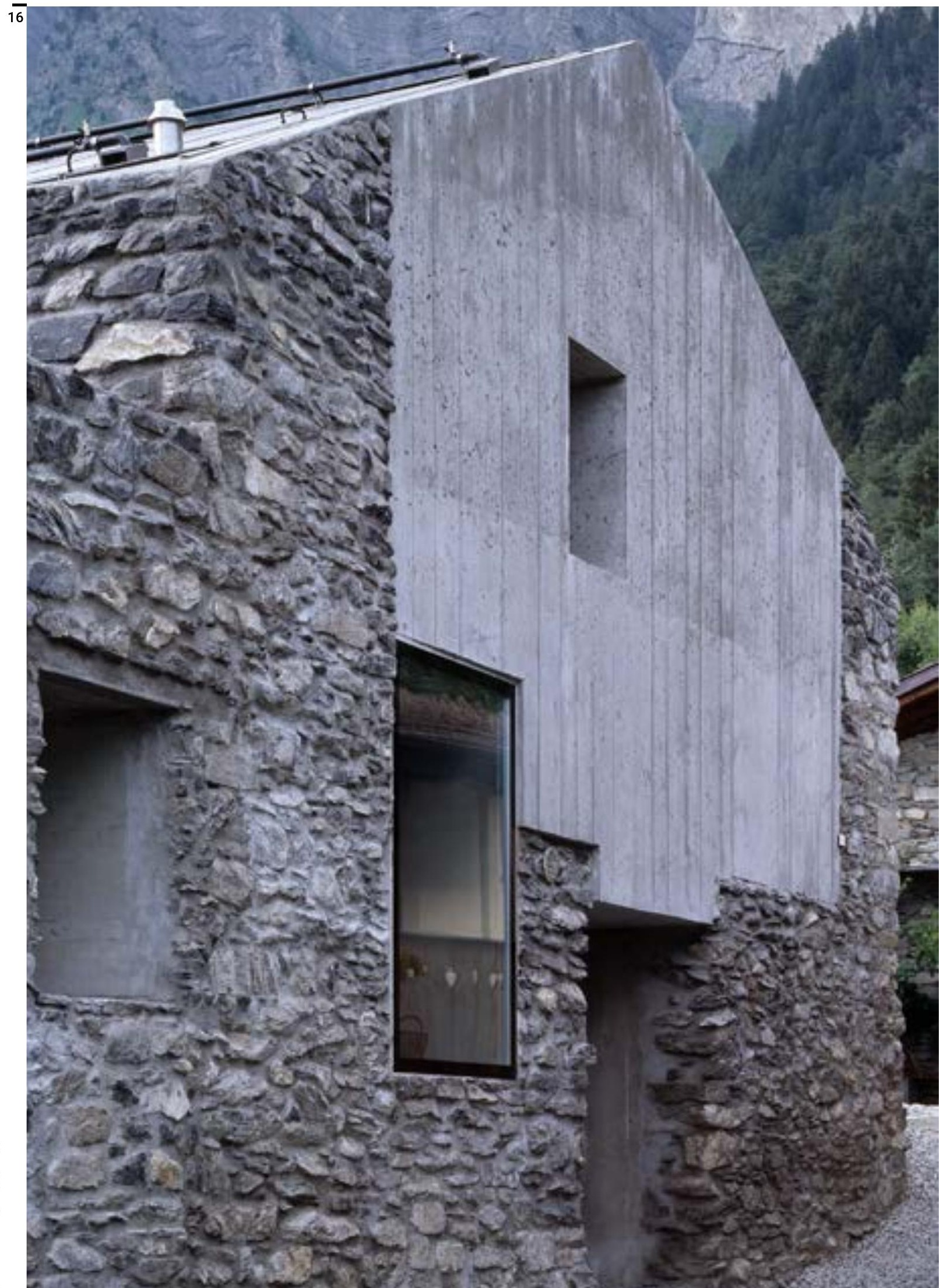

Fig. 16

Casa Roduit,

\section{$\overline{16}$}




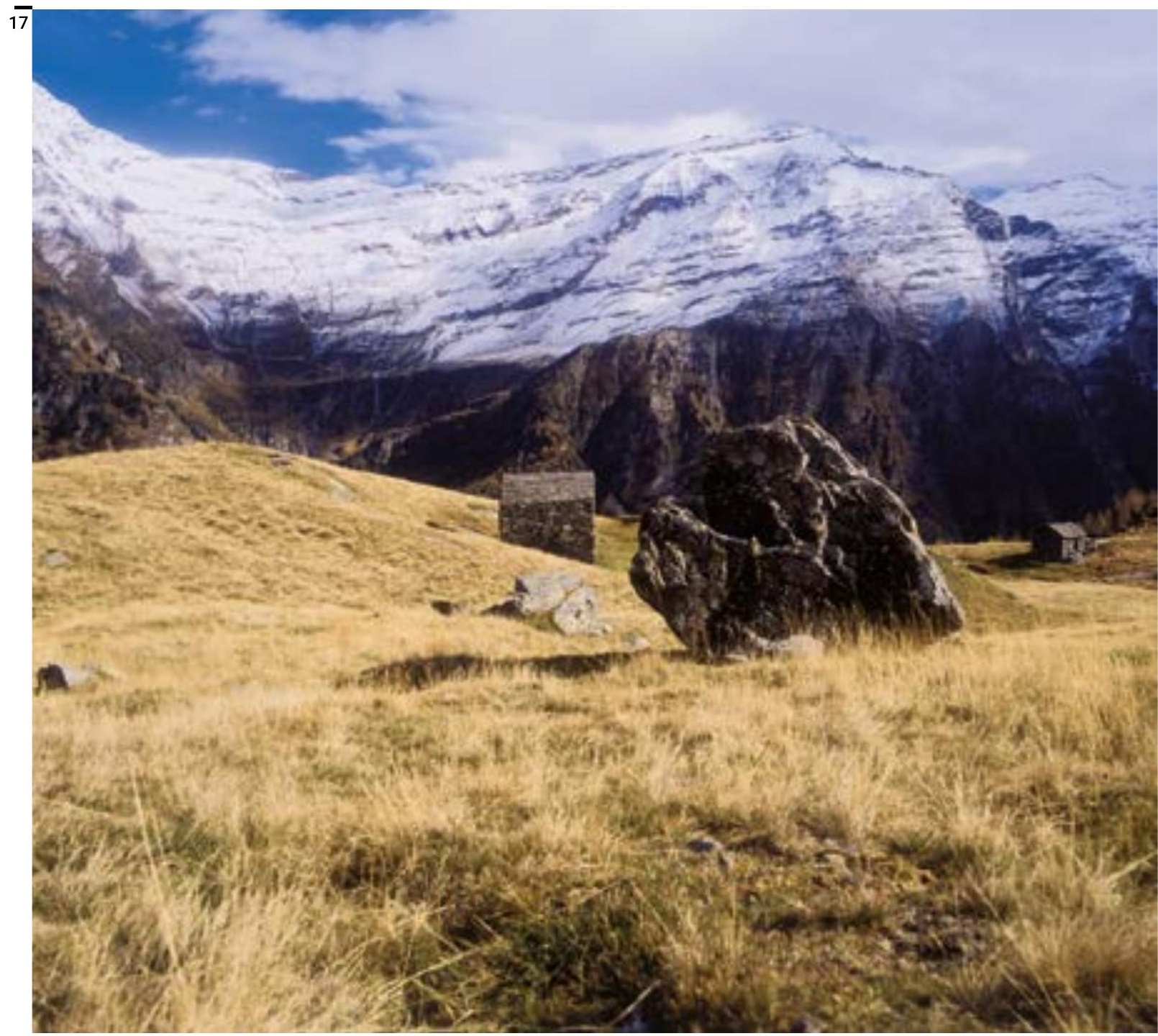

Fig. 17

Martino Pedrozzi,

Sceru, 2002 (foto

Pino Brioschi).

Fig. 18

18

Progetto di recupero

del cimitero storico

di Timau-Cleulis,

Ceschia e Mentil

Architetti Associati,

2007-2009, Timau-

Cleulis, Udine.

Figg. 19-20

Casa Savioz, La

Giète-Délé, Vallese,

Savioz Fabrizzi,

2009-2013 (foto

Thomas Jantscher).

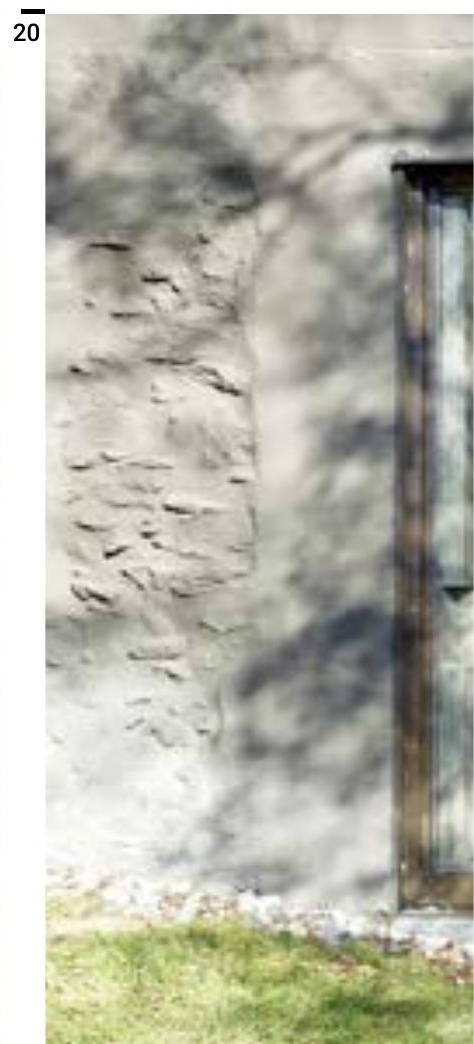



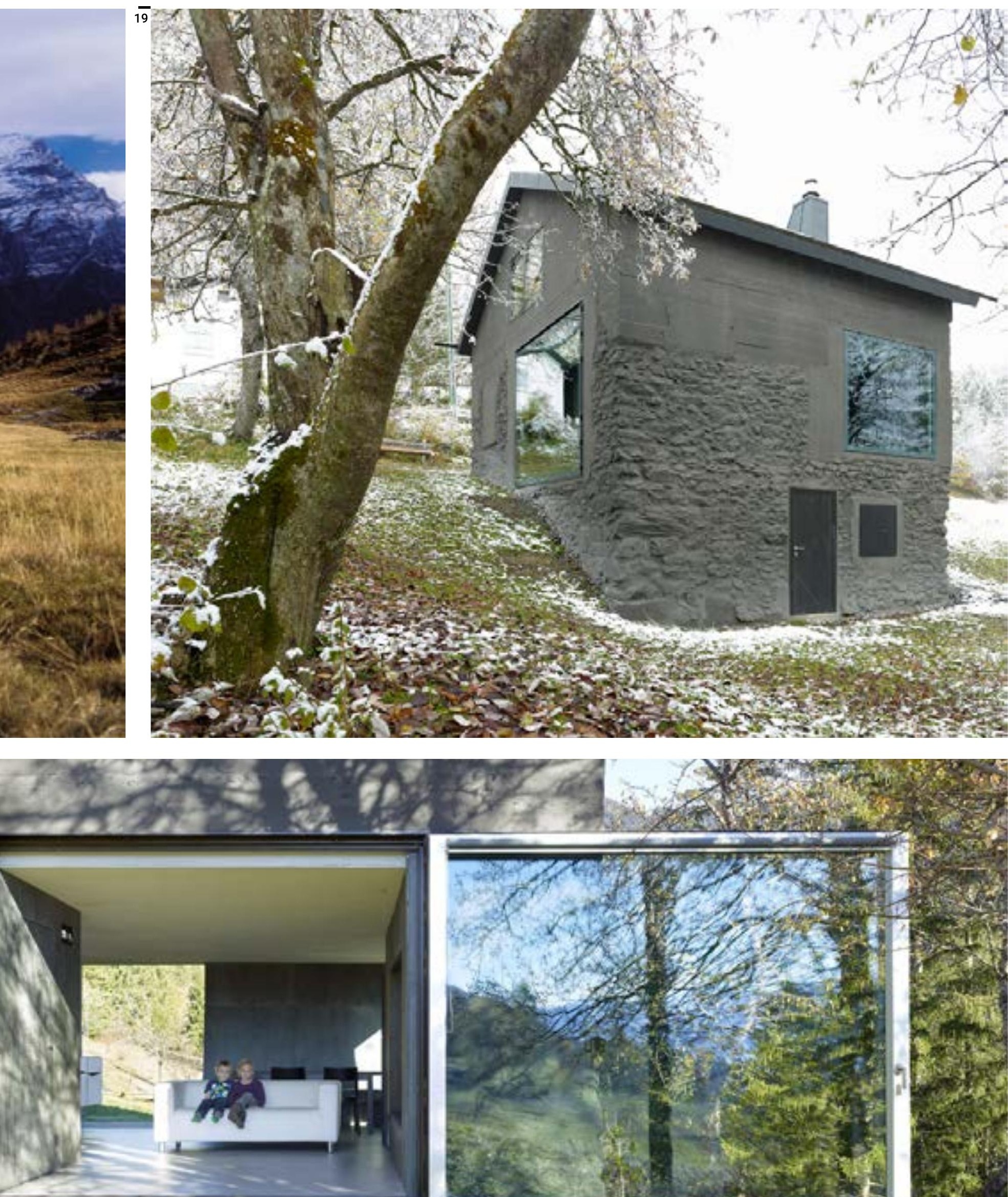

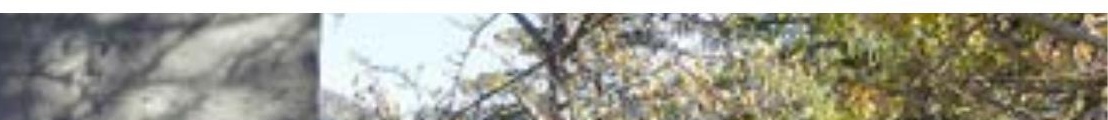

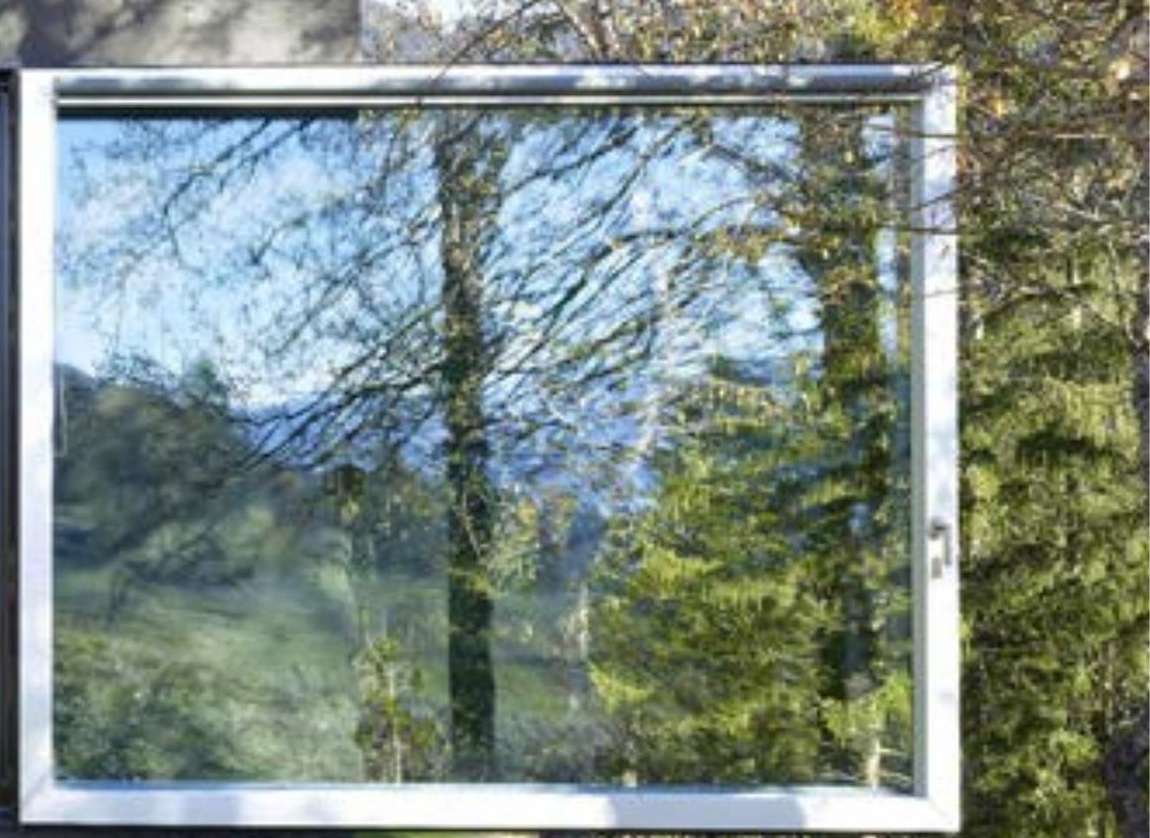


Fig. 21-22

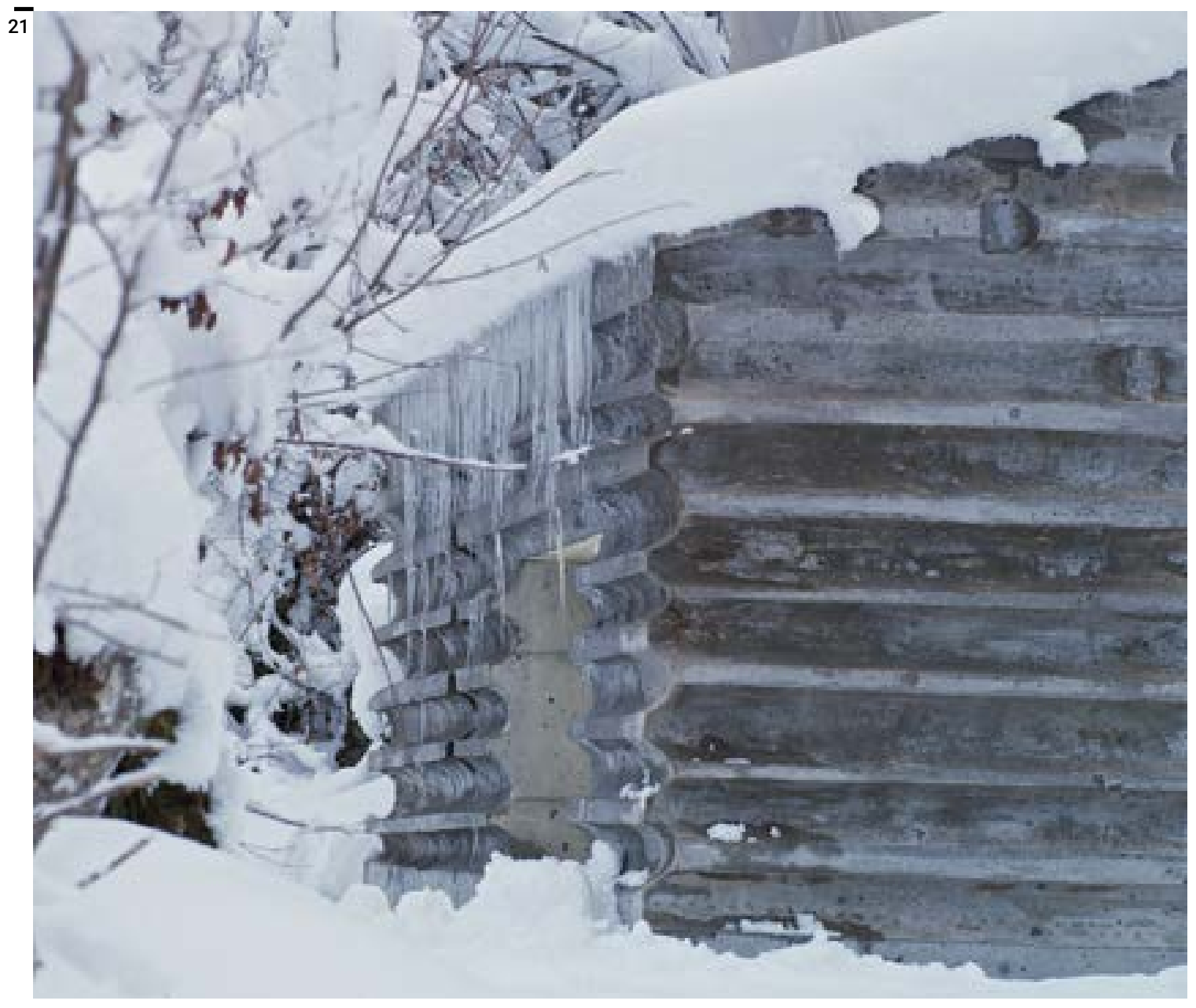

Flims, Nickisch Sano

Walder Architects,

2014 (foto Gaudenz

Danuser)

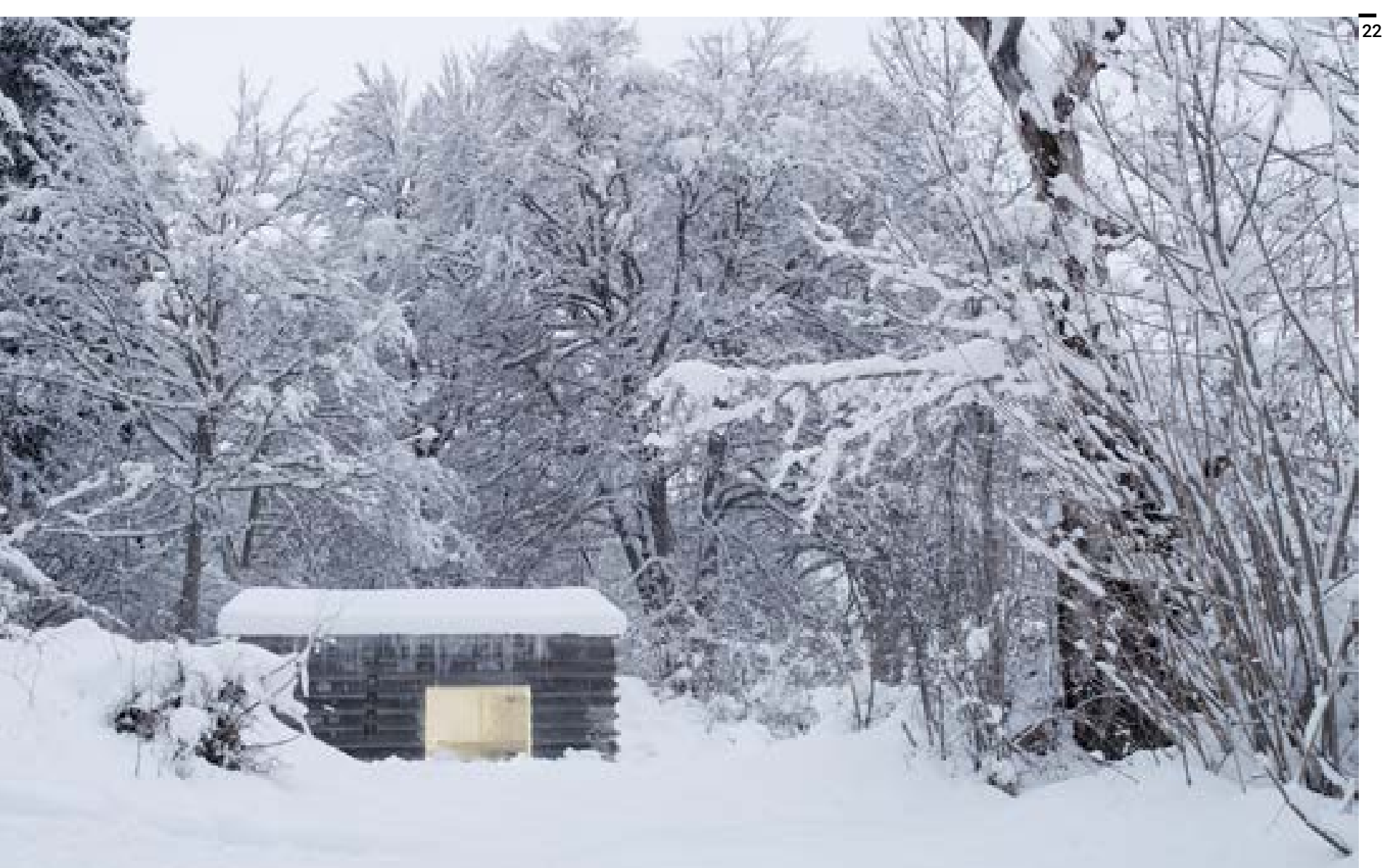


sformarli in una forma astratta e plastica, facendo una vera e propria restituzione del manufatto edilizio all'ambiente circostante.

Alcuni progetti infine fanno un richiamo alle caratteristiche architettonico-formali o costruttive del patrimonio edilizio alpino attraverso uno slittamento semantico che mantiene la memoria del manufatto attraverso la reinterpretazione stilistica, concettuale ed estetizzante delle sue peculiarità architettoniche.

Nel caso della Concrete house di Nickisch Sano Walder Architects a Flims il sistema del blockbau e le caratteristiche materiche delle travi in legno rimangono impresse in negativo nel getto in calcestruzzo armato che costituisce l'involucro esterno del piccolo edificio.

\section{Bibliografia}

Bätzing Werner (2005), Le Alpi. Una regione unica al centro dell'Europa, Bollati Boringhieri, Torino.

Crettaz Bernard (2011), Disneyalp. Riflessioni di un etnografo conservatore museale alpino, Priuli \& Verlucca, Scarmagno (TO)

Crettaz Bernard (1993), «Dieci punti per reinterpretare una scoperta», in COTRAO (a cura di), L'uomo e le Alpi, Vivalda, Quart (AO).

Heinich Nathalie (2009), La fabrique du patrimoine. De la cathédrale à la petite cuillère, Maison des Sciences de l'Homme, coll. «Ethnologie de la France», Paris.

Olmo Carlo (2013), Architettura e storia. Paradigmi della discontinuità, Donzelli, Roma.

Simmel Georg (1911), «La Rovina», trad. it. Carchia Gianni (1981), Estetica delle Rovine, in Rivista di Estetica, n. 8. Steinmann Martin (1996), «Découvrir le monde des choses», in Faces, n. 38. 